September 28, $2018 \quad 16: 25$ WSPC/INSTRUCTION $\quad$ FILE

\title{
THE QUANTUM-CLASSICAL TRANSITION: THE FATE OF THE COMPLEX STRUCTURE
}

\author{
G. MARMO* \\ Dip. Sc. Fisiche and INFN-Napoli, Universitá Federico II, Via Cintia \\ Napoli, 80125, Italy \\ marmo@na.infn.it \\ G. Scolarici \\ Dip. Fisica and INFN-Lecce, Universitá di Lecce, Via per Arnesano \\ Lecce, 73100, Italy \\ scolarici@le.infn.it \\ A. Simoni \\ Dip. Sc. Fisiche and INFN-Napoli, Universitá Federico II, Via Cintia \\ Napoli, 80125, Italy \\ simoni@na.infn.it \\ F. Ventriglia \\ Dip. Sc. Fisiche and INFN-Napoli, Universitá Federico II, Via Cintia \\ Napoli, 80125, Italy \\ ventriglia@na.infn.it \\ Received (Day Month Year) \\ Revised (Day Month Year)
}

\begin{abstract}
According to Dirac, fundamental laws of Classical Mechanics should be recovered by means of an "appropriate limit" of Quantum Mechanics. In the same spirit it is reasonable to enquire about the fundamental geometric structures of Classical Mechanics which will survive the appropriate limit of Quantum Mechanics. This is the case for the symplectic structure. On the contrary, such geometric structures as the metric tensor and the complex structure, which are necessary for the formulation of the Quantum theory, may not survive the Classical limit, being not relevant in the Classical theory. Here we discuss the Classical limit of those geometric structures mainly in the Ehrenfest and Heisenberg pictures, i.e. at the level of observables rather than at the level of states. A brief discussion of the fate of the complex structure in the Quantum-Classical transition in the Schroedinger picture is also mentioned.
\end{abstract}

Keywords: Quantum Mechanics; Geometric Structures; Operator Algebra.

*Dip. Sc. Fisiche, Universitá Federico II, Via Cintia 80126 Napoli, Italy. 
September 28 bedlevo atti $04^{\circ}$ sissa

\section{Introduction}

According to Dirac [1]:

"Classical mechanics must be a limiting case of quantum mechanics. We should thus expect to find that important concepts in classical mechanics correspond to important concepts in quantum mechanics and, from an understanding of the general nature of the analogy between classical and quantum mechanics, we may hope to get laws and theorems in quantum mechanics appearing as simple generalizations of well known results in classical mechanics".

In the Dirac approach to Quantum Mechanics, the carrier space is a Hilbert space, i.e. it is a vector space endowed with a Hermitian Structure which give rise to a Riemannian Structure (the real part of the inner product), a symplectic structure (the imaginary part of the inner product) and a connecting (1-1)-tensor, the complex structure $J$ satisfying $J^{2}=-1$.

On the other hand in Classical Mechanics the prevailing structure is provided by the symplectic structure, it is therefore a natural question to ask what happens, in the appropriate limit as suggested by Dirac, of both the complex and Riemannian structures.

Of course a similar question can be asked with respect to the linear structure. Here, however, the situation is more involved because, due to the probabilistic interpretation of Quantum Mechanics, one requires that only the probability density is physically meaningful and not the probability amplitude, this means that from the measurement view point, the carrier space of Quantum Mechanics is the complex projective space associated with $\mathbb{H}$ rather than $\mathbb{H}$ itself. On this manifold there is still a Kähler structure [2] but the linear structure has disappeared, showing that it is not the linear structure which matters but rather a superposition (composition rule) for solutions, allowing to construct new solutions out of two given ones, as it is well known this phenomenon is well understood in the framework of Riccati-type equations and we will not insist any further on the subject, we refer to the existing literature [3] on the argument.

As for Quantum Mechanics, there has been a recent proposal to define a superposition rule on pure states [4] (the complex projective space of $\mathbb{H}$ ) which amounts to the introduction of a connection on the complex projective space. This connection is essentially given by the Pancharatnam phase, which is also known in the literature as the Berry phase or geometric phase. [5]

Apart from the details concerning the linear structure or the superposition principle of Quantum Mechanics, the Kähler structure is always present and it is a challenge to understand what happens of the complex structure when the appropriate limit to go from Quantum to Classical is taken. Because we want to consider the fate of the complex structure in the Quantum-Classical transition, it is more appropriate to deal directly with a real vector space and a complex structure $J$ defined on it, rather than consider the Hilbert space and hide the complex structure in the defining properties of the space itself. 
Before entering our discussion we should point out that the role played by the complex structure in Quantum Mechanics was already considered by H. Reichenbach in his book "Philosophic Foundations of Quantum Mechanics" .[6] It was also extensively considered by Stueckelberg and his collaborators.[7] Afterwards we find comments by G.W. Mackey [8] and a more extensive treatment by V. Cantoni. [9]

A decade ago a new analysis started [10] also in connection with a proposal by S. Weinberg [11] on a non-linear Quantum Mechanics.

In referring to previous papers we have not distinguished between papers actually focused on the role of the complex structure and those dealing with the role of the Riemannian structure, the reason being that for us the symplectic structure is not questioned because (as a structure) it survives the "appropriate limit" to Classical Mechanics. Therefore, with a symplectic structure granted, the complex structure and the Riemannian structure are not independent and each one determines the other one.

Because we are mostly interested in the Classical limit, we are obliged to take into account various pictures of Quantum Mechanics to consider the Classical limit separately for each one of them. Traditionally, one considers the Schroedinger picture and the Heisenberg picture, in which the equations of motion are considered on the space of states or on the algebra of observables respectively. Here we would like to replace the Heisenberg picture with what we may call the Ehrenfest picture, that is we deal with quadratic functions, expectations values, rather than with operators.

\section{Indetermination relations: the need of a complex structure}

In this section we discuss in a slightly generalized form the quoted argument of Stueckelberg which shows the necessity of introducing a complex structure $J$ to get indetermination relations and formulate Quantum Mechanics. Therefore we start with a real Hilbert space .

An indetermination relation $\grave{a} l a$ Heisenberg usually is a relation like

$$
\left\langle\Delta A^{2}\right\rangle_{\Psi}\left\langle\Delta B^{2}\right\rangle_{\Psi} \geq\langle i[A, B]\rangle_{\Psi}^{2}
$$

where the error operators of the observables $A$ and $B$

$$
\Delta A=A-\langle A\rangle_{\Psi} ; \Delta B=B-\langle B\rangle_{\Psi},
$$

are related to their commutator through the Hermitian operator $i[A, B]$.

However, on a real Hilbert space, the multiplication by the imaginary unit $i$ in $i[A, B]$ does not make sense. Moreover, $[A, B]$ is a skew-Hermitian operator, so that its mean value with respect to a real scalar product always vanishes.

Then, motivated by scaling arguments, in principle there are two possibilities :

$$
\left\langle\Delta A^{2}\right\rangle_{\Psi}\left\langle\Delta B^{2}\right\rangle_{\Psi} \geq\left\{\begin{array}{l}
\mu^{2}\left\langle-[A, B]^{2}\right\rangle_{\Psi} \\
\mu^{2}\langle K(A, B)\rangle_{\Psi}^{2}
\end{array}\right.
$$

where $\mu$ is a real number and $K(A, B)$ a Hermitian bilinear expression to be determined . 
September $\quad 28$

FILE

G Marmo, G Scolarici, A Simoni, F Ventriglia

The first possibility leads to a contradiction for bounded operators unless $\mu=0$ :

In fact, choosing $\Psi$ in an orthonormal basis $\left\{\Phi_{n}\right\}$ in which $A$ is diagonal: $\left\langle\Delta A^{2}\right\rangle_{\Psi}=0$ and $\left\langle\Delta B^{2}\right\rangle_{\Psi}$ is bounded if $B$ is bounded, so their product vanishes while on the contrary $\left\langle-[A, B]^{2}\right\rangle_{\Psi}=\sum_{n}\left(A_{\Psi}-A_{n}\right)^{2}\left\langle\Psi|B| \Phi_{n}\right\rangle^{2}$ is strictly positive.

Only the existence of a complex structure $J$ leads to an indetermination relation:

An indetermination relation (inequality) holds on a real Hilbert space for Hermitian operators $A, B$ (observables) commuting with a complex structure $J$. It has the form:

$$
\left\langle\Delta A^{2}\right\rangle_{\Psi}\left\langle\Delta B^{2}\right\rangle_{\Psi} \geq \frac{1}{4}\langle J[A, B]\rangle_{\Psi}^{2}
$$

The following is a detailed proof which shows that a generalization is possible. Consider the following real scalar product:

$$
\langle(\Delta A+\lambda X \Delta B) \Psi,(\Delta A+\lambda X \Delta B) \Psi\rangle \geq 0,
$$

where $\lambda$ is a real number and $X$ is an operator which has to be determined in such a way that an indetermination relation holds. Expanding the preceding expression leads to

$$
\left\langle\Delta A^{2}\right\rangle_{\Psi}+\lambda^{2}\left\langle\Delta B X^{\dagger} X \Delta B\right\rangle_{\Psi}+\lambda\left\langle\Delta B X^{\dagger} \Delta A+\Delta A X \Delta B\right\rangle_{\Psi} \geq 0
$$

therefore the condition is obtained:

$$
X^{\dagger} X=1
$$

The expression is positive for every value of $\lambda$ when:

$$
\left\langle\Delta A^{2}\right\rangle_{\Psi}\left\langle\Delta B^{2}\right\rangle_{\Psi} \geq \frac{1}{4}\langle K\rangle_{\Psi}^{2}
$$

where

$$
K=\Delta A X \Delta B+\Delta B X^{\dagger} \Delta A
$$

If a complex structure $J$ is available, we may choose

$$
X:=\cos \theta+J \sin \theta \Longrightarrow X^{\dagger} X=1
$$

and look for the maximum of $\langle K(\theta)\rangle_{\Psi}^{2}$.

We have

$$
\langle K(\theta)\rangle_{\Psi}=\left\langle[\Delta A, \Delta B]_{+}\right\rangle_{\Psi} \cos \theta+\langle J[A, B]\rangle_{\Psi} \sin \theta=: \alpha \cos \theta+\beta \sin \theta
$$

Then

$$
\frac{d}{d \theta}\langle K(\theta)\rangle_{\Psi}^{2}=0 \Longrightarrow \tan \theta_{\max }=\frac{\beta}{\alpha} \Longrightarrow\left\langle K_{\max }\right\rangle_{\Psi}^{2}=\alpha^{2}+\beta^{2}
$$

so that eventually

$$
\left\langle\Delta A^{2}\right\rangle_{\Psi}\left\langle\Delta B^{2}\right\rangle_{\Psi} \geq \frac{1}{4}\left\langle[\Delta A, \Delta B]_{+}\right\rangle_{\Psi}^{2}+\frac{1}{4}\langle J[A, B]\rangle_{\Psi}^{2}
$$


This general form of the indetermination relation, first discovered by Schroedinger [12] and Robertson [13] in the 30's, reduces to the weaker Heisenberg relation for uncorrelated states (see also [14]).

Remark The same expression for the indetermination relation holds also on the complexified (via $J$ ) Hilbert space.

\section{The Schroedinger picture}

Before we have discussed the need of a complex structure $J$ in Quantum Mechanics. In this section we discuss the relevant geometric structures which appear in Quantum Mechanics and the relations among them.

To avoid technicalities, for the time being, while we deal with general aspects, we shall confine ourselves to finite dimensional carrier spaces; later, to consider the transition to Classical Mechanics, we will deal with operators acting on infinite dimensional Hilbert spaces.

We consider a vector space $V$ equipped with a complex structure $J$, that is a (1-1)-tensor with the property $J^{2}=-1$. As $V$ is a vector space its linear structure determines -and is determined by- a vector field $\Delta$, the infinitesimal generator of dilation (often it is also called the Liouville vector field or the Euler operator).

If we introduce Cartesian coordinates for $V$, say $\left\{x^{j}\right\}$, we have

$$
\Delta=x^{j} \frac{\partial}{\partial x^{j}}
$$

while for the complex structure we find

$$
J=J_{k}^{j} d x^{k} \otimes \frac{\partial}{\partial x^{j}}
$$

with the property

$$
J_{k}^{j} J_{m}^{k}=-\delta_{m}^{j} .
$$

The existence of $J$ on the vector space $V$ implies that $\operatorname{dim} V$ is even, say $2 n$. We denote by $g$ the metric tensor (Euclidean metric tensor on $V$ ) defined by

$$
g=g_{j k} d x^{j} \otimes d x^{k}
$$

with det $\left\|g_{j k}\right\| \neq 0$ and $g_{j k} x^{j} x^{k}$ a positive-definite quadratic function.

The admissibility condition of $g$ and $J$ is stated [15] by requiring that

$$
\begin{gathered}
g(J v, J w)=g(v, w), \\
g(J v, w)+g(v, J w)=0
\end{gathered}
$$

i.e. $J$ is an orthogonal transformation both at the finite level (Eq.(5)) and at the infinitesimal level (Eq.(6)).

Out of $g$ and $J$ we may construct a symplectic structure $\omega$ defined by

$$
\omega(v, w)=g(J v, w) .
$$


September $\quad 28$

FILE

G Marmo, G Scolarici, A Simoni, F Ventriglia

It follows that $\omega$ is not degenerate and

$$
\omega(v, w)=-\omega(w, v)
$$

that is $\omega$ is indeed a symplectic structure.

Having these four structures on $V$, we may consider the group of diffeomorphisms of $V$ and identify subgroups by requiring that one or more of the previous structures are preserved.

By requiring that $\varphi_{*} \Delta=\Delta$ we collapse the infinite dimensional diffeomorphism group to the finite dimensional general linear group $G L(V)$.

The requirement $\varphi^{*} \omega=\omega$ provides us with the infinite dimensional subgroup of symplectic (canonical) transformations. While $\varphi^{*} g=g$ gives us the finite dimensional rotation group and $\varphi^{*} J=J$ identifies the general linear group $G L(n, \mathbb{C})$.

By taking the intersection of any two linear subgroups associated with any pair of admissible structures we get the subgroup of unitary transformations with infinitesimal generators provided by the antiHermitian operators with respect to the Hermitian structure

$$
h(v, w):=g(v, w)+i \omega(v, w)=:<v \mid w>.
$$

We recall that an Hermitian structure on $V$, as a real vector space, can be defined as a map:

$$
h: V \times V \longrightarrow \mathbb{R}^{2}
$$

such that

$$
h(v, w) \longrightarrow(g(v, w), \omega(v, w)) .
$$

We can also induce a complex structure on $V$ by setting, for $z=(\alpha+i \beta) \in \mathbb{C}$ and $u \in V$,

$$
z u=(\alpha+i \beta) u=\alpha u+J(\beta u) .
$$

To consider the product structure on functions and more specifically on quadratic functions, it is convenient to consider directly the target space $\mathbb{R}^{2}$, in Eq.(10), as $\mathbb{C}$, the complex numbers. In this way, if $V$ is considered as a real vector space,

$$
h(v, w)=g(v, w)+i w(v, w)
$$

is a complex valued quadratic function of real variables.

\section{Remarks}

i) Notice that if the admissibility condition of Eq.(5) does not hold, we can always build a Hermitian structure out of a given $g$ by substituting it with the symmetrized metric tensor:

$$
g_{s}(., .)=: \frac{1}{2}\{g(J ., J .)+g(., .)\} .
$$

which will be positive and nondegenerate if $g$ is. 
September $\quad 28$

FILE

Quite similarly [16], if the relation of Eq.(7) does not define a complex structure $J$, that is if $\left(g^{-1} \omega\right)^{2} \neq-\mathbb{I}$, then Riesz's theorem tells us that there exists a nonsingular linear operator $A$ such that:

$$
\omega(x, y)=g(A x, y)
$$

and the antisymmetry of $\omega$ implies:

$$
g(A x, y)=-g(x, A y)
$$

i.e. that $A$ is skew-hermitian: $A^{\dagger}=-A$, and: $-A^{2}>0$. Let then $P$ be a (symmetric) nonnegative square root of $-A^{2}$. $P$ will be injective, so $P^{-1}$ will be well defined ${ }^{\mathrm{a}}$. We define then: $J=: A P^{-1}$ and:

$$
g_{\omega}(., .)=: g(P(.), .)
$$

Therefore:

$$
\omega(x, y)=g(A x, y)=g_{\omega}(J x, y)
$$

and: $J^{\dagger}=-J, J^{2}=-\mathbb{I}$. The triple $\left(g_{\omega}, J, \omega\right)$ will be then an admissible triple, Eq.(6) will hold true for $g_{\omega}$ and, moreover:

$$
g_{\omega}(J x, J y)=g(A x, J y)=-g(A J x, y)=g_{\omega}(x, y)
$$

and Eq.(5) will be satisfied as well.

ii) The adjoint $A^{\dagger}$ of any linear operator $A$ with respect to a metric tensor $g$ is defined by the standard relation:

$$
g\left(A^{\dagger} x, y\right)=: g(x, A y)
$$

and we can read Eq.(5) as saying that the complex structure $J$ is skew-adjoint with respect to the metric tensor $g$.

Although it may seem elementary, it is worth stressing here that, despite the fact that we are working in a real vector space, the adjoint of $A$ does not coincide with the transpose $A^{T}$ for a general $g$. Indeed, spelling out explicitly Eq.(19) in terms of matrices leads to:

$$
A^{\dagger}=g^{-1} A^{T} g
$$

and therefore, even for real matrices: $A^{\dagger}=A^{T}$ only if the metric is standard Euclidean one and, in general, symmetric matrices need not be self-adjoint.

To completely turn entities depending on the linear structure on the space of states into tensorial objects, we notice that with every matrix $A \equiv\left\|A_{k}^{j}\right\| \in \mathfrak{g l}(2 n, \mathbb{R})$ we can associate both a $(1-1)$-tensor

$$
T_{A}=A_{k}^{j} d x^{k} \otimes \frac{\partial}{\partial x^{j}}
$$

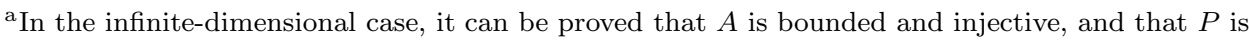
also injective and densely defined, so that $P^{-1}$ is well defined in the infinite-dimensional case as well.
} 
and a linear vector field

$$
X_{A}=A_{k}^{j} x^{k} \frac{\partial}{\partial x^{j}}
$$

The two are connected by

$$
T_{A}(\Delta)=X_{A}
$$

and are both homogeneous of degree zero, i.e.

$$
L_{\Delta} X_{A}=L_{\Delta} T_{A}=0 .
$$

The correspondence $A \longrightarrow T_{A}$ is a full associative algebra and a corresponding Lie algebra isomorphism. The correspondence $A \longrightarrow X_{A}$ is instead only a Lie algebra (anti)isomorphism, that is

$$
T_{A} \circ T_{B}=T_{A B}
$$

while

$$
\left[X_{A}, X_{B}\right]=-X_{[A, B]} .
$$

Moreover, for any $A, B \in \mathfrak{g l}(2 n, \mathbb{R})$ :

$$
L_{X_{A}} T_{B}=-X_{[A, B]} .
$$

Out of the Liouville vector field $\Delta$ and the metric tensor $g$, we can construct the quadratic function

$$
\mathfrak{g}=\frac{1}{2} g(\Delta, \Delta)
$$

along with the associated Hamiltonian vector field $\Gamma$ via the

$$
i_{\Gamma} \omega=-d \mathfrak{g} .
$$

It is possible to show that

$$
\Gamma=J(\Delta)
$$

or $J(\Gamma)=-\Delta$.

The vector field $\Gamma$ preserves all three structures $g, \omega$ and $J$. Thus the vector field $\Gamma$ will be a generator of the unitary group and may be associated with a Schroedinger-type equation

$$
i \hbar \frac{d}{d t} \Psi=L_{\Gamma} \Psi=H \Psi
$$

where we have used the more familiar notation $\Psi \in V$ instead of $u \in V$.

More generally, starting from Eqs.(28), (29) and (30), we can construct quadratic functions associated with any linear vector field $X_{A}$ by defining

$$
f_{A}=\frac{1}{2} g\left(\Delta, X_{A}\right) .
$$


Then again we have

$$
i_{Y_{A}} \omega=-d f_{A}
$$

where now

$$
Y_{A}=J\left(X_{A}\right)
$$

whenever the matrix $A$ satisfies the condition of the following Eq.(36).

In general, any vector field $X_{A}$ will define the equations of motion of a Quantum systems if

$$
L_{X_{A}} \omega=0 ; L_{X_{A}} g=0
$$

and, a fortiori, $L_{X_{A}} J=0$.

This implies that the matrix $A$, representative of the vector field, satisfies the relations

$$
\omega A=(\omega A)^{t r} \Longleftrightarrow \omega A+A^{t r} \omega=0
$$

while the condition $L_{X_{A}} g=0$ implies that

$$
g A+(g A)^{t r}=g A+A^{t r} g=0,
$$

where, with abuse of notation, we have used $\omega$ and $g$ to represent a matrix associated with the corresponding tensors.

By using the vector fields $\Delta$ and $J(\Delta)$, which are an involutive distribution, i.e. in particular they commute, we get out of $V-\{0\}$ the complex projective space associated with the Hilbert space structure on $V$ defined by the Hermitian form $h$ (see Eq.(9)), which will be denoted by $\mathbb{P}(V)$.

By construction, the unitary group acting on $V$ in terms of linear transformation will induce an action on $\mathbb{P}(V)$ which turns out to be a transitive action.

The first outcome of our description in terms of tensors is that now instead of linear operators on $\mathbb{P}(V)$ we can use vector fields on $\mathbb{P}(V)$ which are autonomously defined without making recourse to the underlined vector space $V$. The main point here is that $\mathbb{P}(V)$ is not a linear space therefore it makes no sense to consider on it linear operators, one has to induce an action from linear operators acting on $V$.

The use of vector fields allows to bypass the construction of the action in terms of $V$.

\section{The momentum map associated with the symplectic action of the unitary group}

We have remarked that the linear action of the unitary group on $V$ is provided by the intersection of the symplectic group and the rotation group.

Because the group acts symplectically, there will be an associated momentum map from the vector space to the dual of the Lie algebra, say

$$
\mu: V \longrightarrow \mathfrak{u}^{*}(n)
$$


September $\quad 28$

FILE

in explicit terms we have

$$
\mu(v)=i|v><v|, \quad v \in V
$$

where we have used the bra and ket notation of Dirac, and identified $\mathfrak{u}^{*}(n)$ with $\mathfrak{u}(n)$ through a trace operation. By using our previous structures, it would be

$$
\mu(v)=i v h(v, .)
$$

which acts on $\mathfrak{u}(n)$ in the following way:

$$
\mu(v)(A)=i<v|A| v>
$$

for any $A \in \mathfrak{u}(n)$.

To have a momentum map equivariant with respect to the action of $\Delta$ and $J(\Delta)$ we may set

$$
\widetilde{\mu}(v)=i \frac{|v><v|}{<v \mid v>}
$$

or

$$
\widetilde{\mu}(v)=i \frac{v h(v, .)}{h(v, v)} .
$$

This map represents the momentum map associated with the symplectic action of $U(n)$ on the complex projective space $\mathbb{P}(V)$.

It is now clear that all vector fields associated with Hermitian operators on $V$ will pass to the quotient $\mathbb{P}(V)$ because they commute with $\Delta$ and preserve $J$.

With any Hermitian operator $A$ we associate $X_{A}$ which projects onto $\mathbb{P}(V)$, giving a symplectic vector field with generating function

$$
\tilde{f}_{A}(\Psi)=\frac{<\Psi \mid A \Psi>}{<\Psi \mid \Psi>}
$$

that is

$$
\tilde{f}_{A}(\Psi)=\frac{h(\Psi, A \Psi)}{h(\Psi, \Psi)}
$$

We observe that functions here defined may be expressed through the quadratic functions defined by Eq.(32) of the previous Section: they are nothing but the quadratic functions projected onto $\mathbb{P}(V)$

$$
\tilde{f}_{A}(\Psi)=\frac{<\Psi \mid A \Psi>}{<\Psi \mid \Psi>}=\frac{g\left(\Delta, X_{A}\right)}{g(\Delta, \Delta)}
$$

It is now possible to formulate the equations of motion in terms of this "mean values" of the Hermitian operators. 
September . 28

2018 16:25 WSPC/INSTRUCTION

FILE

\section{The Ehrenfest picture}

The Ehrenfest picture [17] of Quantum Mechanics originates from the Ehrenfest theorem. We may formulate it within our approach in the following way.

We consider the Lie algebra of the unitary group realized in terms of skewHermitian operators on $V$ and we define a map

$$
E: \mathfrak{u}(n) \times V \longrightarrow \mathbb{R} \quad ; \quad(\imath A, v) \longrightarrow \frac{h(v, A v)}{h(v, v)}=\tilde{f}_{A}(v)
$$

This evaluation map has the property that

$$
\widetilde{E}: V \longrightarrow \operatorname{Lin}(\mathfrak{u}(n), \mathbb{R}) \equiv \mathfrak{u}^{*}(n)
$$

coincides with the momentum map associated with the symplectic action of the unitary group acting on $V$.

Our aim now is to realize the algebra of linear operators on $V$ in the Ehrenfest picture, that is by means of quadratic functions. We observe that the definition of Eq.(32) in Sec.(3)is not suitable to this end, because the real scalar product $g$ annihilates the skew-symmetric part of any operator $A$. Therefore, we have to use the Hermitian form $h$ defined by Eq.(9) of Sec.(3) and deal with complex-valued functions of real variables:

$$
f_{A}(x)=\frac{1}{2} h(A x, x) ; x \in V .
$$

This definition reduces to the previous one and yields real functions for Hermitian operators, while associates imaginary functions to skew-hermitian operators, so that it realizes a one to one correspondence between operators and complex-valued quadratic functions. We notice that neither $g$ nor $\omega$ separately can be used to recover the full associative algebra: in fact $\omega$ allows to recover the Lie algebra structure of the Hermitian operators, but it is not able to recover the symmetrized product on Hermitian operators, i.e. the Jordan algebra structure [18] existing on the space of Hermitian operators. To recover this product we have to use the metric tensor $g$. It is only the use of the entire admissible triple $(g, \omega, J)$ or the equivalent Hermitian structure $h$ that allows to give a complete description of the full algebra of operators in the Ehrenfest scheme.

We now introduce two brackets on quadratic functions corresponding respectively to Lie and Jordan operator algebras and from them recover the associative operator product.[10] In fact, it is well known that on the symplectic vector space $(V, \omega)$ we can define a Poisson tensor $\Lambda$ which is the "inverse" of $\omega$ : in coordinates we have

$$
\Lambda=\Lambda^{j k} \frac{\partial}{\partial x^{j}} \wedge \frac{\partial}{\partial x^{k}}
$$

with $\Lambda^{j k} \omega_{k m}=\delta_{m}^{j}$.

The associated Poisson Bracket is given by

$$
\left\{f_{1}, f_{2}\right\}:=\Lambda\left(d f_{1}, d f_{2}\right)=\Lambda^{j k} \frac{\partial f_{1}}{\partial x^{j}} \frac{\partial f_{2}}{\partial x^{k}} .
$$


September $\quad 28$

FILE

On quadratic functions we have the following result:

$$
\left\{f_{A}, f_{B}\right\}(x)=f_{J[A, B]}(x)=-i f_{[A, B]}(x) .
$$

In fact, supposing $A, B$ Hermitian operators, in coordinates

$$
f_{A}=\frac{1}{2} g_{m l} A_{n}^{l} x^{n} x^{m} ; f_{B}=\frac{1}{2} g_{s p} B_{t}^{p} x^{t} x^{s} .
$$

Then, bearing in mind that

$$
\Lambda^{k j} g_{j m}=-J_{m}^{k}
$$

we have:

$$
\begin{gathered}
\Lambda^{j k} \frac{\partial f_{A}}{\partial x^{j}} \frac{\partial f_{B}}{\partial x^{k}}=\Lambda^{j k}(A x)_{j}(B x)_{k}=\Lambda^{j k} g_{m j} g_{n k}(A x)^{m}(B x)^{n}= \\
=-\frac{1}{2} g_{m j} J_{n}^{j}(B x)^{n}(A x)^{m}+\frac{1}{2} g_{n k} J_{m}^{k}(A x)^{m}(B x)^{n}= \\
\quad=\frac{1}{2} \omega([A, B] x, x)=-\frac{i}{2} h([A, B] x, x)=-i f_{[A, B]}(x) .
\end{gathered}
$$

Moreover

$$
f_{[A, B]}(x)=\frac{1}{2} h([A, B] x, x)=\frac{i}{2} \omega([A, B] x, x)=\frac{i}{2} g(J[A, B] x, x)=i f_{J[A, B]}(x)
$$

The proof is analogous in the cases when one or both operators $A$ and $B$ are skew-symmetric, due to the relation $\omega=g J$.

By using in a similar way the inverse of the metric tensor $g=g_{i k} d x^{j} \otimes d x^{k}$, say

$$
G=G^{j k} \frac{\partial}{\partial x^{j}} \otimes \frac{\partial}{\partial x^{k}}
$$

with $G^{j k} g_{k m}=\delta_{m}^{j}$, we may define a new Bracket (we shall call it the RiemannJordan Bracket) on functions as:

$$
\left(f_{1}, f_{2}\right):=G\left(d f_{1}, d f_{2}\right)=G^{j k} \frac{\partial f_{1}}{\partial x^{j}} \frac{\partial f_{2}}{\partial x^{k}} .
$$

By using quadratic functions we get the relation

$$
\left(f_{A}, f_{B}\right)(x)=f_{(A B+B A)}(x) .
$$

In fact, assuming again $A, B$ Hermitian operators, and bearing in mind that $G^{k j} g_{j m}=\delta_{m}^{k}$, we have in coordinates

$$
\begin{gathered}
G^{j k} \frac{\partial f_{A}}{\partial x^{j}} \frac{\partial f_{B}}{\partial x^{k}}=G^{j k}(A x)_{j}(B x)_{k}=G^{j k} g_{m j} g_{n k}(A x)^{m}(B x)^{n}= \\
=\frac{1}{2} g_{n k} \delta_{m}^{k}(A x)^{m}(B x)^{n}+\frac{1}{2} g_{m j} \delta_{n}^{j}(B x)^{n}(A x)^{m}=\frac{1}{2} g\left([A, B]_{+} x, x\right)=
\end{gathered}
$$




$$
=\frac{1}{2} h\left([A, B]_{+} x, x\right)=f_{(A B+B A)}(x) .
$$

Finally, we introduce an associative product on quadratic functions as

$$
\left(f_{A} * f_{B}\right)(x):=f_{A B}(x)
$$

so that

$$
\left(f_{A} * f_{B}\right)(x)+\left(f_{B} * f_{A}\right)(x)=f_{(A B+B A)}(x)=\left(f_{A}, f_{B}\right)(x)
$$

and

$$
\left(f_{A} * f_{B}\right)(x)-\left(f_{B} * f_{A}\right)(x)=f_{(A B-B A)}(x)=i\left\{f_{A}, f_{B}\right\}(x),
$$

so we obtain the following result:

$$
\left(f_{A} * f_{B}\right)(x)=\frac{1}{2}\left(f_{A}, f_{B}\right)(x)+\frac{i}{2}\left\{f_{A}, f_{B}\right\}(x),
$$

which is the analog of the operator decomposition $A B=\frac{1}{2}[A, B]_{+}+\frac{1}{2}[A, B]$.

It is also apparent that the following relation which connects gradient and Hamiltonian fields holds (see Eq.(34) of Sec.(3)):

$$
\operatorname{grad} f_{A}:=G d f_{A}=-J \Lambda d f_{A} .
$$

Equations of motion in this picture have the Classical-like form

$$
\hbar \frac{d}{d t} f_{A}=\left\{f_{H}, f_{A}\right\}
$$

Within the one-to-one correspondence between operators and quadratic functions, we recover the Heisenberg equations of motion in Hamiltonian form.

We conclude this section by observing that the $*-$ product is reminiscent of the Weyl-Wigner approach and the Moyal Bracket. It shows also that a full isomorphism of algebras between quadratic functions and operators requires the introduction of a non-local product.

Summarizing, we have replaced the initial description provided by Dirac in terms of Hilbert spaces and operators acting on them with a description on the differentiable manifold of pure states (the complex projective space) in terms of vector fields and functions. It is clear that if we do not have a linear structure we have no meaning of quadratic functions, therefore the "image" of quadratic functions on $V$ shall be identified autonomously.

This identification is achieved by requiring that Hamiltonian vector fields associated with them should be Killing vectors with respect to the metric structure on $\mathbb{P}(V)$ arising from the Kähler structure. This ends up being the infinitesimal version of Wigner's theorem.[19] 
September $\quad 28$

FILE

\section{The Classical Limit}

To consider the Quantum-Classical transition, the best way is to consider a description which uses the same carries space for both Classical and Quantum descriptions, i.e. smooth functions on the phase space. The Classical picture uses the point-wise product, while the Quantum picture uses the $*$-product. In particular we shall restrict to a phase-space which is a symplectic vector space and the $*$-product is the Moyal product.

Given a symplectic vector space $(E, \omega)$, a Weyl system is defined to be a strongly continuous map from $E$ to unitary transformations on some Hilbert space $\mathbb{H}$ :

$$
W: E \rightarrow U(\mathbb{H})
$$

with

$$
W\left(e_{1}\right) W\left(e_{2}\right) W^{\dagger}\left(e_{1}\right) W^{\dagger}\left(e_{2}\right)=e^{\frac{i}{\hbar} \omega\left(e_{1}, e_{2}\right)} \mathbb{I},
$$

with $\mathbb{I}$ the identity operator.

Consider a Lagrangian subspace $L$ and an associated isomorphism

$$
E \rightleftharpoons L \oplus L^{*}=T^{*} L .
$$

On $L$ we consider square integrable functions with respect to a Lebesgue measure

on $L$, a measure invariant under translations. The splitting of $E$ allows to define $e=(\alpha, x)$ and set

$$
\begin{gathered}
W((0, x) \Psi)(y)=\Psi(x+y), \\
W((\alpha, 0) \Psi)(y)=e^{i \alpha(y)} \Psi(y), \\
x, y \in L, \alpha \in L^{*}, \Psi \in L^{2}\left(L, d^{n} y\right) ;
\end{gathered}
$$

it is obvious that $W(e)$ are unitary operators and moreover they satisfy Weyl condition of Eq.(2) with $\omega$ being the canonical one on $T^{*} L$.

The strong continuity allows to use Stone's theorem to get infinitesimal generators $R(e)$ such that

$$
W(e)=e^{i R(e)} \quad \forall e \in E
$$

and $R(\lambda e)=\lambda R(e)$ for any $\lambda \in \mathbb{R}$.

When we select a complex structure on $E$

$$
J: E \rightarrow E \quad, \quad J^{2}=-1,
$$

we may define "creation" and "annihilation" operators by setting

$$
\begin{array}{r}
a(e)=\frac{1}{\sqrt{2}}(R(e)+i R(J e)), \\
a^{\dagger}(e)=\frac{1}{\sqrt{2}}(R(e)-i R(J e)) .
\end{array}
$$


September $\quad 28$

FILE

By using this complex structure on $E$ we may construct an inner product on $E$ as

$$
\left\langle e_{1}, e_{2}\right\rangle=\omega\left(J e_{1}, e_{2}\right)-i \omega\left(e_{1}, e_{2}\right),
$$

therefore creation and annihilation operators are associated with a Kähler structure on $E$.

The Weyl map can be extended to functions on $T^{*} L \rightleftharpoons E$; indeed we first define the symplectic Fourier transform of $\hat{f} \in \mathfrak{F}(E)$

$$
f(q, p)=\frac{1}{2 \pi \hbar} \int \hat{f}(\alpha, x) e^{\frac{i}{\hbar}(\alpha q-x p)} d \alpha d x
$$

and then associate with it the operator $\widehat{A}_{f}$ defined by

$$
\widehat{A}_{f}=\frac{1}{2 \pi \hbar} \int \hat{f}(\alpha, x) e^{\frac{i}{\hbar}(\alpha \widehat{Q}-x \widehat{P})} d \alpha d x
$$

Vice versa, with any operator $A$ acting on $\mathbb{H}$ we associate a function $f_{A}$ on the symplectic space $E$ by setting

$$
f_{A}(e):=\operatorname{Tr} A W(e) ;
$$

this map is called the Wigner map and, via a symplectic Fourier transform, is the inverse of the Weyl map. A new product of functions may be introduced on $\mathfrak{F}(E)$ by setting

$$
\left(f_{A} \star f_{B}\right)(e):=\operatorname{Tr} A B W(e)=f_{A B}(e) .
$$

We thus find that a symplectic structures on $E$ give rise to an associative product on $\mathfrak{F}(E)$, which is not commutative.

The dynamics on $\mathfrak{F}(E)$ can be written in terms of this non-commutative product as

$$
i \hbar \frac{d f_{A}}{d t}=f_{H} \star f_{A}-f_{A} \star f_{H}
$$

Having clarified the setting, let us consider a very simple situation to actually carry on the limiting procedure.

Without any loss of generality we may limit to consider a bidimensional symplectic vector space

$$
\mathbb{R}^{2}=\mathbb{R} \oplus \mathbb{R} ; \omega=d x \wedge d y .
$$

The map $\hat{A} \mapsto f_{A}$ given by $f_{A}(x, y)=\operatorname{Tr} \hat{A} W(x, y)$ induces a Moyal product

$$
\left(f_{A} \star f_{B}\right)(x, y):=\operatorname{Tr} \hat{A} \hat{B} W(x, y) .
$$

By using the specific form of $W(x, y)$ it is possible to provide an explicit form in terms of bidifferential operators. We have

$$
\left(f_{A} \star f_{B}\right)(x, y)=f_{A}(x, y) \exp \left[i \frac{\hbar}{2}\left(\frac{\overleftarrow{\partial}}{\partial x} \frac{\vec{\partial}}{\partial y}-\frac{\overleftarrow{\partial}}{\partial y} \frac{\vec{\partial}}{\partial x}\right)\right] f_{B}(x, y)
$$


where a standard notation for physicists has been used, i.e. $\overleftarrow{\partial_{x}}$ and $\overrightarrow{\partial_{y}}$ mean that the operators act on the left or on the right, respectively.

Alternatively, we may write

$$
\left(f_{A} \star f_{B}\right)(x, y)=\exp \left[i \frac{\hbar}{2} \mathfrak{L}\right] f_{A}\left(x^{\prime}, y^{\prime}\right) f_{B}(x, y)
$$

where the bidifferential operator $\mathfrak{L}$ has been introduced

$$
\mathfrak{L}:=\left[\frac{\partial}{\partial x^{\prime}} \frac{\partial}{\partial y}-\frac{\partial}{\partial y^{\prime}} \frac{\partial}{\partial x}\right]_{x^{\prime}=x, y^{\prime}=y}
$$

The action of $\mathfrak{L}$ may be expressed in terms of the Poisson Bracket of two functions $f, g \in \mathfrak{F}\left(\mathbb{R}^{2}\right)$ as:

$$
\begin{gathered}
\mathfrak{L} f g=f_{x} g_{y}-f_{y} g_{x}=:\{f, g\} \\
\mathfrak{L}^{2} f g=\mathfrak{L}\{f, g\}=\left\{f_{x}, g_{y}\right\}-\left\{f_{y}, g_{x}\right\}=f_{x x} g_{y y}-2 f_{x y} g_{y x}+f_{y y} g_{x x}
\end{gathered}
$$

and in general (all $\partial$ 's commute among themselves)

$$
\mathfrak{L}^{n+1} f g=\mathfrak{L}^{n}\{f, g\}=\sum_{k=0}^{n}\left(\begin{array}{l}
n \\
k
\end{array}\right)(-1)^{k}\left\{\partial_{x}^{n-k} \partial_{y}^{k} f, \partial_{y}^{n-k} \partial_{x}^{k} g\right\}
$$

so that

$$
\left(f_{A} \star f_{B}\right)(x, y)=f_{A}(x, y) f_{B}(x, y)+i \frac{\hbar}{2}\left\{f_{A}, f_{B}\right\}+\frac{1}{2}\left(i \frac{\hbar}{2}\right)^{2} \mathfrak{L}^{2} f_{A} f_{B}+\ldots
$$

from which we see that the Quantum corrections to the point-wise Abelian product are expressed by means of the complex structure times the Planck's constant and the Poisson Bracket of the two functions and of all their derivatives.

Now, even or odd powers of $\mathfrak{L}$ are respectively symmetric or skewsymmetric with respect to the exchange of $f_{A}$ and $f_{B}$, so that the commutator of $f_{A}$ and $f_{B}$ contains only the odd powers of $\mathfrak{L}$ :

$$
f_{A} \star f_{B}-f_{B} \star f_{A}=2 i \frac{\hbar}{2}\left[\left\{f_{A}, f_{B}\right\}+\frac{1}{6}\left(i \frac{\hbar}{2}\right)^{2} \mathfrak{L}^{3} f_{A} f_{B}+\ldots\right]
$$

and the Quantum equation of motion of $f_{B}$ with respect to the Hamiltonian $f_{H}$ reads

$$
i \hbar \frac{d}{d t} f_{B}=f_{H} \star f_{B}-f_{B} \star f_{H}=i \hbar\left[\left\{f_{H}, f_{B}\right\}+O\left(\hbar^{2}\right)\right]
$$

and finally, dropping terms $O\left(\hbar^{2}\right)$, we recover the Classical equations of motion in the Hamiltonian form:

$$
\frac{d}{d t} f_{B}^{\circ}=\left\{f_{H}, f_{B}^{\circ}\right\}
$$

When the Hamiltonian $f_{H}$ is a quadratic polynomial, the $O\left(\hbar^{2}\right)$ terms vanish, so this result is exact and we recover the Ehrenfest's theorem. 
September $\quad 28$

2018 16:25 WSPC/INSTRUCTION

FILE

This "appropriate Classical limit" shows very clearly that while in Quantum Mechanics the associative and non commutative product among functions determines all the various structures, i.e. the complex structure and the Poisson bracket, because the Lie product and therefore the Jordan product are uniquely determined by the operator-product [20], in the Classical picture the point-wise product does not fix a unique "compatible Poisson bracket". Indeed all possible Poisson tensors on phase space define derivations for the point-wise product on functions on the phase space.

To exhibit more clearly this aspect, i.e. the role of the symmetric tensor in the definition of the $*$-product we use a slightly modified product with respect to the one given by Moyal. This generalization arises from the fact that one may give a different, more general, definition of Weyl systems where "different ordering procedures" are considered instead of the fully symmetrized one provided by the Weyl prescription.

It is the following one [21]

$$
W\left(e_{1}\right) W\left(e_{2}\right) W^{\dagger}\left(e_{1}\right) W^{\dagger}\left(e_{2}\right)=e^{-g\left(e_{1}, e_{2}\right)+i \omega\left(e_{1}, e_{2}\right)} \mathbb{I} .
$$

In analogy with this modification we may consider a $*$-product given by

$$
\left(f_{A} \star f_{B}\right)(x, y)=\exp \frac{\lambda}{2}[\mathfrak{G}+i \mathfrak{L}] f_{A}\left(x^{\prime}, y^{\prime}\right) f_{B}(x, y)
$$

where the bidifferential operator $\mathfrak{G}$ has been introduced:

$$
\mathfrak{G}:=\left[\frac{\partial}{\partial x^{\prime}} \frac{\partial}{\partial x}+\frac{\partial}{\partial y^{\prime}} \frac{\partial}{\partial y}\right]_{x^{\prime}=x, y^{\prime}=y} .
$$

and we have used a dimensionless deformation parameter $\lambda$ instead of $\hbar$.

Now we get

$\left(f_{A} \star f_{B}\right)(x, y)=f_{A}(x, y) f_{B}(x, y)+\frac{\lambda}{2}\left(\partial_{x} f_{A} \partial_{x} f_{B}+\partial_{y} f_{A} \partial_{y} f_{B}\right)+i \frac{\lambda}{2}\left\{f_{A}, f_{B}\right\}+O\left(\lambda^{2}\right)$.

We consider the Classical limit of the Lie product and the Classical limit of the Jordan product to find

$$
\begin{gathered}
\frac{1}{2}\left(f_{A} \star f_{B}-f_{B} \star f_{A}\right)(x, y)=i \frac{\lambda}{2}\left\{f_{A}, f_{B}\right\}+O\left(\lambda^{2}\right), \\
\frac{1}{2}\left(f_{A} \star f_{B}+f_{B} \star f_{A}\right)(x, y)=f_{A}(x, y) f_{B}(x, y)+\frac{\lambda}{2}\left(\partial_{x} f_{A} \partial_{x} f_{B}+\partial_{y} f_{A} \partial_{y} f_{B}\right)+O\left(\lambda^{2}\right)(
\end{gathered}
$$

respectively.

We see immediately that if the product should reduce to the commutative pointwise product the symmetric bidifferential operator cannot be there.

\section{Conclusions and outlook}

Let us summarize our line of argumentation. In Quantum Mechanics the product of operators (observables) uniquely defines the Lie product compatible with it (i.e. such that it defines derivations of the given product). 


$\begin{aligned} & \text { September } \\ & \text { bedlevo atti 04 sissa }\end{aligned} 28, \quad 2018 \quad 16: 25 \quad$ WSPC/INSTRUCTION $\quad$ FILE

If we associate operators with functions (a necessary replacement if we want to deal with physical pure states, points of the complex projective space), the product among operators induces a $\star$-product on functions. In the Weyl-Wigner approach this product can be expressed by means of bidifferential operators (generated by a Poisson tensor in the case of the Weyl ordering and by a Poisson and a metric tensors in the case of normal or anti-normal ordering).

In the Classical limit, the product gives rise to a point-wise product which does not privilege any specific Poisson Bracket. However, a Poisson Bracket may be selected by considering the Classical limit of the Lie product. A similar Classical limit for the Jordan product would anyway start with the point-wise term and therefore would not give rise naturally to a symmetric tensor on phase-space.

Our argument has been presented without privileging any dynamical system. It is however true that if we consider special systems like the Harmonic Oscillator, by considering the limit of operators realized in terms of creation and annihilation operators, we would get a Classical phase-space described in terms of complex coordinates. However, these would not exist for generic Hamiltonian systems.

As a further comment, we may add that the Classical limit may be also considered in the Schroedinger picture.

Here the the Classical limit would require first that the operators are realized as differential operators on some "configuration space", acting on square integrable functions defined on the same configuration space.

The Classical limit would correspond to the replacement of the differential operators with their symbols [17] and the complex structure would appear only at the level of the so called "Quantum potential" having $\hbar^{2}$ as coefficient. Moreover, out of the coupled equations for the real and the imaginary part of the wave function, we would obtain only one equation (for $\hbar^{2} \rightarrow 0$ ) represented by the Hamilton-Jacobi equation. A complete solution for this equation would allow for the construction of a solution for the other one (the transport equation).[22]

Therefore, having obtained uncoupled equations in the Classical limit, the complex structure is not required to couple them as in the Quantum situation.

We shall further elaborate on these points somewhere else.

\section{References}

[1] P.A.M. Dirac, The Principles of Quantum Mechanics, 4th Edition (Pergamon, Oxford, 1958).

[2] A. Weil, Introduction à l'Etudes Variétés Kähleriennes (Hermann, Paris, 1958).

[3] J.F. Cariñena, J. Grabowski and G. Marmo, Lie-Scheffers Systems: A geometric Approach (Bibliopolis, Napoli, 2000).

[4] V.I.Mank'o, G. Marmo, E.C.G. Sudarshan, F. Zaccaria, Interference and entanglement: an intrinsic approach, J. Phys. A: Math. and Gen. 35 (2002), 7137-7157.

[5] S. Pancharatnam, Generalized theory of interference, and its applications, Proc. Ind. Acad. Sci. A 44 (1956), 247-262.

[6] H. Reichenbach Philosophic Foundations of Quantum Mechanics (University of California Press, Berkeley and Los Angeles, 1944). 
September $\quad 28$

2018 16:25 WSPC/INSTRUCTION

FILE

[7] E.C.G. Stueckelberg, Quantum Theory in Real Hilbert Space, Helv Phys Acta 33 (1960), 727-751;

E.C.G. Stueckelberg and M. Guenin, Quantum Theory in Real Hilbert Space II (Addenda and Errata), Helv Phys Acta 34 (1961), 621.

[8] G. W. Mackey, Mathematical Foundations of Quantum Mechanics (Benjamin, New York, 1963);

G. W. Mackey, Induced Representations of Groups and Quantum Mechanics (Benjamin, New York, 1968).

[9] V. Cantoni, Generalized "Transition Probability", Commun. Math. Phys. 44 (1975), 125-128;

V. Cantoni, The Riemannian Structure on the States of Quantum-like Systems, Commun. Math. Phys. 56 (1977), 189-193;

V. Cantoni,Intrinsic geometry of the quantum-mechanical "phase space", hamiltonian systems and Correspondence Principle, Lincei-Rend. Sc. fis. mat. e nat. LXII (1977), 628-636.

[10] R. Cirelli, A. Manià and L. Pizzocchero, Quantum Mechanics as an infinitedimensional Hamiltonian systems with uncertainty structure: Part I, J. Math. Phys. 31 (1990), 2891-2897; R. Cirelli, A. Manià and L. Pizzocchero, Quantum Mechanics as an infinite-dimensional Hamiltonian systems with uncertainty structure: Part II, J. Math. Phys. 31 (1990), 2898-2903.

[11] S. Weinberg, Testing quantum Mechanics, Ann. Phys. 194 (1989), 336-386;

S. Weinberg, Precision tests of Quantum Mechanics,Phys. Rev. Lett. 62 (1989), 485488;

S. Weinberg, Weinberg replies,Phys. Rev. Let. 63 (1989), 1115.

[12] E. Schroedinger, Zum Heisenbergshen Unshärfeprinzip, Ber. Kgl. Akad. Wiss.296(1930), 296-303.

[13] H.P. Robertson, A general formulation of the uncertainty principle and its classical interpretation, Phys. Rev. 35 (1930), 667;

H.P. Robertson, An Indeterminacy Relation for Several Observables and Its Classical Interpretation, Phys. Rev. 46 (1934), 794-801.

[14] E.C.G. Sudarshan, C.B. Chiu, and G. Bhamati, Generalized uncertainty relations and characteristic invariance for the multimode states, Phys. Rev. A 52 (1995), 43-54.

[15] G. Marmo, G. Morandi, A. Simoni and F. Ventriglia, Alternative structures and biHamiltonian systems, J.Phys. A: Math.Gen. 35 (2002), 8393-8406;

A. Canas da Silva, Lectures on Symplectic Geometry (Springer, Berlin, 2001).

[16] R. Abraham and J. E. Marsden, Foundations of Mechanics, $2^{\text {nd }}$ Ed. (Addison-Wesley, Reading, 1985).

[17] G. Esposito, G. Marmo and G. Sudarshan, From Classical to Quantum Mechanics (Cambridge University Press, Cambridge, 2004).

[18] P. Jordan, J. von Neumann, E. Wigner, On an algebraic generalization of the quantum mechanical formalism, Ann. Math. 35 (1934), 29-64.

[19] E. P. Wigner, Group theory and its application to the quantum Mechanics of atomic spectra (Academic Press, New York-London, 1959)

[20] J. Grabowski, G. Marmo, Binary operations in classical and quantum Mechanics, in J. Grabowski, P. Urbanski (eds.), Classical and Quantum integrabiblity, Banach Center Publ. 59 , 163-172 (2003).

[21] P. Aniello, V.I. Mank'o, G. Marmo,S. Solimeno,F. Zaccaria On the coherent states, displacement operators and quasidistributions associated with deformed quantum oscillator, Jour. of Optics B 2(2000), 718-725.

[22] V.A. Fock, Fundamentals of Quantum Mechanics (MIR, Moscow, 1978) 